\title{
On the relationship between $L$-fuzzifying approximation spaces and $L$-fuzzifying pretopological spaces
}

\author{
Anand P. Singh and I. Perfilieva \\ University of Ostrava, Institute for Research and Applications of Fuzzy Modeling \\ NSC IT4Innovations, 30. dubna 22, 70103 \\ Ostrava 1, Czech Republic, \\ anandecc@gmail.com, irina.perfilieva@osu.cz
}

\begin{abstract}
The aim of this contribution is to establish the interrelationship between $L$-fuzzifying approximation spaces based on reflexive $L$ fuzzy relations and $L$-fuzzifying pretopological spaces. This connection is established in the category theoretic setup.
\end{abstract}

Keywords: Concrete category; $L$-fuzzifying approximation space; $L$-fuzzifying pretopological space; Galois connection; Alexandroff $L$-fuzzifying topology.

\section{Introduction}

The concept of rough set was firstly proposed by Pawlak [11]. This theory attracted many researchers due to its importance in the study of intelligent systems with insufficient and incomplete information. In past decades the theory developed significantly because of usefulness in variety of applications. Several generalizations of rough sets have been made by replacing the equivalence relation by an arbitrary relation. After the introduction of fuzzy rough set by Dubois and Prade [3], various interesting studies has been carried on relating the theory of fuzzy rough sets with fuzzy topologies (cf., $[2,7,13,16,17,18]$ ). Further, Ying [20] introduced a logical approach to study the fuzzy topology and proposed the notion of fuzzifying topology. A number of articles published based on this new approach (cf., $[4,5,9,19,23,24])$. Fang $[4,5]$ showed the one to one correspondence between fuzzifying topologies and fuzzy preorders and Shi [19] discussed the relationship of fuzzifying topology and specialization preorder in the sense of Lai \& Zhang [8]. In 1999, Zhang [22] studied the fuzzy pretopology through the categorical point of view and Perfilieva et al. in $[12,14]$ discussed its relationship with Ftransform. Further on following the approach of Ying
[20], Lowen and $\mathrm{Xu}[9]$, Zhang [24] discussed the categorical study of fuzzifying pretopology.

In the recent work of Pang [10] $L$-fuzzifying rough sets has been studied through the constructive and axiomatic approaches. So far, the relationship between $L$-fuzzifying pretopological spaces and $L$-fuzzifying approximation spaces has not been studied yet.

This paper is focused on the interrelationship between $L$-fuzzifying approximation spaces based on reflexive $L$-fuzzy relation and $L$-fuzzifying pretopological spaces. It is worth to mention that our motivation is different from [15] in which the connection is established in the sense of [22] rather than $L$-fuzzifying pretopological setting. Moreover, we show that if $(X, \theta)$ is fuzzy preordered based $L$-fuzzifying approximation space then an Alexandroff $L$-fuzzifying topology can be induced.

The structure of the paper is organized as follows. In section 2, we recall some necessary concepts and notations related to category theory, $L$-fuzzy relation and $L$-fuzzifying topology. Section 3 is the main part of this paper. We recall the notion of $L$-fuzzifying approximation spaces and discussed its properties. Further, we introduce the concept of $L$-fuzzifying pretopological spaces and show that it induces a Cech $L$-fuzzy interior operator. Specifically, we established the categorical relationship between $L$-fuzzifying approximation space based on the reflexive $L$-fuzzy relation and $L$-fuzzifying pretopological spaces. Finally, we give the conclusion in section 4 .

\section{Preliminaries}

A De Morgan algebra $\left(L, \vee, \wedge,{ }^{\prime}, 0,1\right)$ is an algebra of type $(2,2,1,0,0)$, where $(L, \vee, \wedge, 0,1)$ is a completely distributive lattice with the least element 0 and greatest element 1 and an order reversing involution " 1 ". Throughout this paper, we consider the membership values from a fixed completely De Morgan algebra $L$. 
Let $X$ be a nonempty set. The set of all subsets of $X$ will be denoted by $\mathcal{P}(X)$. For each $\lambda \in \mathcal{P}(X), \lambda^{c}$ is the complement of $\lambda$ and characteristic function of $\lambda$ is $1_{\lambda}$.

Goguen [6] introduced the notion of $L$-fuzzy sets as a generalization of Zadeh's fuzzy sets. For a nonempty set $X, L^{X}$ denotes the collection of all fuzzy subsets of $X$. Also, for all $a \in L, \mathbf{a}(x)=a$ is a constant $L$ fuzzy set on $X$. The greatest and least element of $L^{X}$ is denoted by $1_{X}$ and $0_{X}$ respectively.

Definition 2.1 [6] Let $X$ be a nonempty set. Then for $\lambda, \mu \in L^{X}$ and for each $x \in X$, the following are induced operations on $L^{X}$ :

(i) $(\lambda \wedge \mu)(x)=\lambda(x) \wedge \mu(x)$,

(ii) $(\lambda \vee \mu)(x)=\lambda(x) \vee \mu(x)$,

(iii) $(\lambda \leq \mu) \Leftrightarrow \lambda(x) \leq \mu(x)$,

(iv) $\lambda=\mu \Leftrightarrow \lambda(x)=\mu(x)$.

Let $I$ be a set of indices, $\lambda_{i} \in L^{X}, i \in I$. The meet and join of elements from $\left\{\lambda_{i} \mid i \in I\right\}$ are defined as follows:

- $\left(\bigwedge_{i \in I} \lambda_{i}\right)(x)=\bigwedge_{i \in I} \lambda_{i}(x), \quad x \in X$,

- $\left(\bigvee_{i \in I} \lambda_{i}\right)(x)=\bigvee_{i \in I} \lambda_{i}(x), \quad x \in X$.

Throughout this paper, all the considered categories are concrete. A concrete category (or construct) [1] is defined over Set. Specifically, it is a pair $(\mathbf{C}, U)$, where $\mathbf{C}$ is a category and $U: \mathbf{C} \rightarrow$ Set is a faithful (forgetful) functor. We say that $U(X)$ is an underlying set for each $\mathbf{C}$-object $\mathrm{X}$. When a forgetful functor is clear from the context, we write only $\mathbf{C}$ instead of pair $(\mathbf{C}, U)$.

A concrete functor between concrete categories $(\mathbf{C}, U)$ and $(\mathbf{D}, V)$ is a functor $F: \mathbf{C} \rightarrow \mathbf{D}$ with $U=V \circ F$. It means, $F$ only changes structures on the underlying sets.

Definition 2.2 [1] Suppose that $F: \mathbf{C} \rightarrow \mathbf{D}, G$ : $\mathbf{D} \rightarrow \mathbf{C}$ are concrete functors. The pair $(F, G)$ is called a Galois connection if either of the following equivalent conditions holds.

(1) $\left\{i d_{Y}: F \circ G(Y) \rightarrow Y \mid Y \in D\right\}$ is a natural transformation from the functor $F \circ G$ to the identity functor on $\mathbf{D}$, and $\left\{i d_{X}: X \rightarrow G \circ F(X) \mid X \in C\right\}$ $i s$ a natural transformation from the identity functor on $\mathbf{C}$ to the functor $G \circ F$.

(2) For each $Y \in \mathbf{D},\left\{i d_{Y}: F \circ G(Y) \rightarrow Y \mid Y \in D\right\}$ is $a \mathbf{D}$ morphism, and for each $X \in \mathbf{C},\left\{i d_{X}: X \rightarrow\right.$ $G \circ F(X) \mid X \in C\}$ is a $\mathbf{C}$ morphism.
If $(F, G)$ is a Galois connection, then it is easy to check that $F$ is a left adjoint of $G$, or equivalently $G$ is a right adjoint of $F$.

Now we recall the following definition of $L$-fuzzy relation from [21].

Definition 2.3 [21] Let $X$ be a nonempty set. An $L$ fuzzy relation $\theta$ on $X$ is a fuzzy subset of $X \times X$. A fuzzy relation $\theta$ is called

(i) reflexive if $\theta(x, x)=1 \forall x \in X$,

(ii) transitive if $\bigvee_{y \in X} \theta(x, y) \wedge \theta(y, z) \leq \theta(x, z)$, $\forall x, y, z \in X$.

$A$ reflexive and transitive $L$-fuzzy relation $\theta$ is called an $L$-fuzzy preorder.

Now, let $f: X \rightarrow Y$ be a map. Then for $\mu \in$ $L^{Y}, f^{-1}(\mu)$ is an inverse image of $\mu$ under $f$, i.e. $f^{-1}(\mu)(x)=\mu(f(x))$.

Definition 2.4 [20] An L-fuzzifying topology $\mathcal{T}$ on universe $X$ is a mapping $\mathcal{T}: \mathcal{P}(X) \rightarrow L$, such that for each $\lambda, \mu \in \mathcal{P}(X),\left\{\lambda_{i} \mid i \in I\right\} \subseteq \mathcal{P}(X)$, the following properties hold:

(i) $\mathcal{T}(\phi)=\mathcal{T}(X)=1$,

(ii) $\mathcal{T}(\lambda \cap \mu) \geq \mathcal{T}(\lambda) \wedge \mathcal{T}(\mu)$

(iii) $\mathcal{T}\left(\bigcup_{i \in I} \lambda_{i}\right) \geq \bigwedge_{i \in I} \mathcal{T}\left(\lambda_{i}\right)$.

For an L-fuzzifying topology $\mathcal{T}$ and nonempty set $X$, the pair $(X, \mathcal{T})$ is called an $L$-fuzzifying topological space.

Further, an L-fuzzifying topological space $(X, \mathcal{T})$ is called Alexandroff, if

(iv) $\mathcal{T}\left(\bigcap_{i \in I} \lambda_{i}\right) \geq \bigwedge_{i \in I} \mathcal{T}\left(\lambda_{i}\right)$.

For two $L$-fuzzifying topological space $\left(X, \mathcal{T}_{X}\right)$ and $\left(Y, \mathcal{T}_{Y}\right)$ a map $f:\left(X, \mathcal{T}_{X}\right) \rightarrow\left(Y, \mathcal{T}_{Y}\right)$ is called continuous if for all $x \in X$ and $\lambda \in \mathcal{P}(Y), \mathcal{T}_{X}\left(f^{-1}(\lambda)\right) \geq$ $\mathcal{T}_{Y}(\lambda)$

\section{$3 \quad L$-fuzzifying approximation space and $L$-fuzzifying pretopology}

In this section, first we remind the notion of $L$ fuzzifying approximation space as it was introduced in [10]. Further, by introducing the notion of $L$-fuzzifying pretopological space, we discuss how to generate an 
$L$-fuzzifying pretopology by a reflexive $L$-fuzzy relation. Our idea is based on the $L$-fuzzifying approximation operator studied in $L$-fuzzifying rough set theory. Moreover, as a categorical viewpoint we establish the Galois connection between the categories of $L$ fuzzifying approximation space and $L$-fuzzifying pretopological space.

Definition 3.1 [10] Let $\theta$ be an L-fuzzy relation on $X$. Then lower $L$-fuzzifying approximation of $\lambda$ is a map $\underline{\theta}: \mathcal{P}(X) \rightarrow L^{X}$ defined by;

$$
\underline{\theta}(\lambda)(x)=\bigwedge_{y \notin \lambda} \theta(x, y)^{\prime}, \quad \forall \lambda \in \mathcal{P}(X), \quad \forall x \in X .
$$

We call $\underline{\theta}$ the lower $L$-fuzzifying approximation operator and the pair $(X, \theta)$ is called an $L$-fuzzifying approximation space based on $L$-fuzzy relation $\theta$.

Remark 3.1 (a) Note that, if $\lambda=X-\{y\} \in \mathcal{P}(X)$ for some $y \in X$, then we have the lower $L$ fuzzifying approximation $\underline{\theta}(X-\{y\})(x)=\theta(x, y)^{\prime}$ for each $x \in X$

(b) The above definition of lower L-fuzzifying approximation operator is a certain reduction of the definition in [10].

In the next proposition we postulate some basic properties of $L$-fuzzifying lower approximation operator, which will be used in the sequel.

Proposition 3.1 [10] Let $(X, \theta)$ be an L-fuzzifying approximation space and $\theta$ be a reflexive $L$-fuzzy relation on $X$. Then for $\lambda \in \mathcal{P}(X)$ and $\left\{\lambda_{i} \mid i \in I\right\} \subseteq$ $\mathcal{P}(X)$, the following holds:

(i) $\underline{\theta}(X)=1_{X}$,

(ii) $\underline{\theta}(\lambda) \leq 1_{\lambda}$,

(iii) $\underline{\theta}\left(\bigcap_{i \in I} \lambda_{i}\right)=\bigwedge_{i \in I} \underline{\theta}\left(\lambda_{i}\right)$.

For given two $L$-fuzzifying approximation spaces $(X, \theta)$ and $(Y, \rho)$, the following is the notion of morphism between them.

Definition 3.2 The morphism $f:(X, \theta) \rightarrow(Y, \rho)$ between two L-fuzzifying approximation spaces $(X, \theta)$ and $(Y, \rho)$ is given by

$$
f^{-1}(\underline{\rho}(\lambda)) \leq \underline{\theta}\left(f^{-1}(\lambda)\right) \quad \forall \lambda \in \mathcal{P}(Y) .
$$

We denote by FYAPP, the category of $L$-fuzzifying approximation space based on reflexive $L$-fuzzy relations and morphisms between them.

Now, we introduce the notion of $L$-fuzzifying pretopological space which is similar (but not identical) to that in [9].
Definition 3.3 An $L$-fuzzifying pretopology on $X$ is a set of functions $\tau_{X}=\left\{p_{x}: \mathcal{P}(X) \rightarrow L \mid x \in X\right\}$ such that for all $\lambda, \mu \in \mathcal{P}(X)$, and $x \in X$,

(i) $p_{x}(X)=1$,

(ii) $p_{x}(\lambda) \leq 1_{\lambda}(x)$,

(iii) $p_{x}(\lambda \cap \mu)=p_{x}(\lambda) \wedge p_{x}(\mu)$.

The pair $\left(X, \tau_{X}\right)$ is called an $L$-fuzzifying pretopological space.

An L-fuzzifying pretopological space $\left(X, \tau_{X}\right)$ is called Alexandroff, if

(iv) $p_{x}\left(\bigcap_{i \in I} \lambda_{i}\right)=\bigwedge_{i \in I} p_{x}\left(\lambda_{i}\right)$.

Note that, for an $L$-fuzzifying pretopological space $\left(X, \tau_{X}\right)$, where $p_{x} \in \tau_{X}$ and $\lambda \in \mathcal{P}(X), p_{x}(\lambda)$ is a degree to which every crisp subset of $X$ i.e., "x belongs to the interior of $\lambda$ ".

The notion of a continuous map between two set endowed with $L$-fuzzifying pretopologies is given below.

Definition 3.4 Let $\left(X, \tau_{X}\right)$ and $\left(Y, \tau_{Y}\right)$ be two $L$ fuzzifying pretopological spaces. Then a map $f$ : $\left(X, \tau_{X}\right) \rightarrow\left(Y, \tau_{Y}\right)$ is called continuous if for all $x \in X$ and $\lambda \in \mathcal{P}(Y)$,

$$
q_{f(x)}(\lambda) \leq p_{x}\left(f^{-1}(\lambda)\right), \text { where } p_{x} \in \tau_{X}, q_{f(x)} \in \tau_{Y} .
$$

Proposition 3.2 The L-fuzzifying pretopological spaces and their continuous maps form a category.

We denote by FYPT, the category of $L$-fuzzifying pretopological space, and if it does not lead to a confusion, the object-class of FYPT will be denoted by FYPT as well.

Let $\tau_{X}=\left\{p_{x}: \mathcal{P}(X) \rightarrow L \mid x \in X\right\}$ be an $L$-fuzzifying pretopology on $X$, then $\tau_{X}$ induces a Cech $L$-fuzzy interior operator $\widehat{i n t}: L^{X} \rightarrow L^{X}$ in a following manner, for all $x \in X, A \in L^{X}$,

$$
\widehat{\operatorname{int}}(A)(x)=\bigvee_{\lambda \in \dot{x}}\left(p_{x}(\lambda) \wedge \bigwedge_{y \in \lambda} A(y)\right)
$$

where, $\dot{x}=\{\mu \in \mathcal{P}(X) \mid x \in \mu\}$.

Proposition 3.3 For every $A, B \in L^{X}$, $a \in L$, the operator $\widehat{i n t}: L^{X} \rightarrow L^{X}$, satisfies the following conditions,
(i) $\widehat{\operatorname{int}}(\mathbf{a})=\mathbf{a}$
(ii) $\widehat{\operatorname{int}}(A) \leq A$, 
(iii) $\widehat{i n t}(A \wedge B)=\widehat{i n t}(A) \wedge \widehat{i n t}(B)$.

Proof: The proof for the properties (i)-(iii) are straight forward and is similar to that of [24].

Proposition 3.4 Suppose that $(X, \theta)$ be an $L$ fuzzifying approximation space and $\theta$ be reflexive $L$ fuzzy relation on $X$. Let for all $\lambda \in \mathcal{P}(X), x \in X$, we denote

$$
p_{x}^{\theta}(\lambda)=\underline{\theta}(\lambda)(x) .
$$

Then $\tau_{\theta}=\left\{p_{x}^{\theta}: \mathcal{P}(X) \rightarrow L \mid x \in X\right\}$, is an L-fuzzifying pretopology on $X$.

Proof: For all $x \in X$ and $\lambda \in \mathcal{P}(X)$, from Proposition 3.1 , it can be easily verified that $\tau_{\theta}$ defined above satisfies the properties (i)-(iii) of lower $L$-fuzzifying approximation operator.

Proposition 3.5 Let $\left(X, \tau_{X}\right)$ be an L-fuzzifying pretopological space. Then for any $x \in X$, we define

$$
\Theta_{\tau_{X}}(x, y)=p_{x}(X-\{y\})^{\prime} .
$$

Then, $\Theta_{\tau_{X}}$ is a reflexive L-fuzzy relation and $\left(X, \Theta_{\tau_{X}}\right)$ is an L-fuzzifying approximation space with reflexive $L$-fuzzy relation $\Theta_{\tau_{X}}$.

Proposition 3.6 If $f:(X, \theta) \rightarrow(Y, \rho)$ is a morphism between two L-fuzzifying approximation spaces, then $f$ is continuous function between two L-fuzzifying pretopological spaces $\left(X, \tau_{\theta}\right)$ and $\left(Y, \tau_{\rho}\right)$.

Proof: The proof follows from Definitions 3.2, 3.4 and Proposition 3.4.

The above proposition gives a concrete functor $\tau:$ FYAPP $\rightarrow$ FYPT between the category of $L$-fuzzifying approximation space and that of $L$ fuzzifying pretopological space.

On the other hand, we prove a proposition, which gives a concrete functor $\Theta:$ FYPT $\rightarrow$ FYAPP i.e. between the category of $L$-fuzzifying pretopological space and that of $L$-fuzzifying approximation space.

Proposition 3.7 If $f$ is a continuous function between two L-fuzzifying pretopological spaces $\left(X, \tau_{X}\right)$ and $\left(Y, \tau_{Y}\right)$. Then $f:\left(X, \Theta_{\tau_{X}}\right) \rightarrow\left(Y, \Theta_{\tau_{Y}}\right)$ is a morphism between two L-fuzzifying approximation spaces.
Proof: Let $\lambda \in \mathcal{P}(Y)$ and $x \in X$, we have

$$
\begin{aligned}
f^{-1}\left(\underline{\Theta_{\tau_{Y}}}(\lambda)\right)(x) & =\frac{\Theta_{\tau_{Y}}(\lambda)(f(x))}{}=\bigwedge_{t \notin \lambda} \Theta_{\tau_{Y}}(f(x), t)^{\prime} \\
& =\bigwedge_{t \notin \lambda} q_{f(x)}(Y-\{t\}) \\
& =\bigwedge_{f(y) \notin \lambda} q_{f(x)}(Y-\{f(y)\}) \\
& \leq \bigwedge_{y \notin f^{-1}(\lambda)} p_{x}\left(f^{-1}(Y-\{f(y)\})\right) \\
& \leq \bigwedge_{y \notin f^{-1}(\lambda)} p_{x}(X-\{y\}) \\
& =\bigwedge_{y \notin f^{-1}(\lambda)} \Theta_{\tau_{X}}(x, y)^{\prime} \\
& =\frac{\Theta_{\tau_{X}}\left(f^{-1}(\lambda)\right)(x) .}{}
\end{aligned}
$$

Hence, we have $f:\left(X, \Theta_{\tau_{X}}\right) \rightarrow\left(Y, \Theta_{\tau_{Y}}\right)$ is a morphism between two $L$-fuzzifying approximation spaces $\left(X, \Theta_{\tau_{X}}\right)$ and $\left(Y, \Theta_{\tau_{Y}}\right)$. In particular, for each $L$ fuzzifying pretopological space $\left(X, \tau_{X}\right), \Theta\left(X, \tau_{X}\right)=$ $\left(X, \Theta_{\tau_{X}}\right)$.

In the next theorem we prove the adjointness between the categories FYAPP and FYPT. Now we have the following.

Proposition 3.8 Let $(X, \theta)$ be an L-fuzzifying approximation space and $\theta$ be reflexive $L$-fuzzy relation. Then $\tau:$ FYAPP $\rightarrow$ FYPT is a left adjoint of $\Theta:$ FYPT $\rightarrow$ FYAPP. Moreover $\Theta \circ \tau(X, \theta)=(X, \theta)$ i.e., $\Theta$ is a left inverse of $\tau$.

Proof: The proof is divided into two parts. On one hand, we show that for any $L$-fuzzifying approximation space $(X, \theta), i d_{X}:(X, \theta) \rightarrow\left(X, \Theta_{\tau_{\theta}}\right)$ is a morphism between $L$-fuzzifying approximation spaces.

For any $\lambda \in \mathcal{P}(X)$ and $x \in X$, we have

$$
\begin{aligned}
\underline{\Theta_{\tau_{\theta}}}(\lambda)(x) & =\bigwedge_{y \notin \lambda} \Theta_{\tau_{\theta}}(x, y)^{\prime} \\
& =\bigwedge_{y \notin \lambda}\left(p_{x}^{\theta}(X-\{y\})^{\prime}\right)^{\prime} \text { (by Prop. 3.5) } \\
& \left.=\bigwedge_{y \notin \lambda} p_{x}^{\theta}(X-\{y\}) \text { (by involution of ' }\right) \\
& \left.=\bigwedge_{y \notin \lambda} \underline{\theta}(X-\{y\})\right) \\
& =\bigwedge_{y \notin \lambda} \theta(x, y)^{\prime} \\
& =\underline{\theta}(\lambda)(x) .
\end{aligned}
$$

Hence, $i d_{X}:(X, \theta) \rightarrow\left(X, \Theta_{\tau_{\theta}}\right)$ is a morphism between $L$-fuzzifying approximation spaces. 
On the other hand, for any $\lambda \in \mathcal{P}(X), x \in X$, we have

$$
\begin{aligned}
p_{x}^{\Theta_{\tau_{X}}}(\lambda) & =\frac{\Theta_{\tau_{X}}}{}(\lambda)(x) \\
& =\bigwedge_{y \notin \lambda} \Theta_{\tau_{X}}(x, y)^{\prime} \\
& =\bigwedge_{y \notin \lambda}\left(p_{x}(X-\{y\})^{\prime}\right)^{\prime} \\
& \left.=\bigwedge_{y \notin \lambda} p_{x}(X-\{y\}) \quad \text { by involution of }^{\prime}\right) \\
& \geq p_{x} \bigcap_{y \notin \lambda}(X-\{y\}) \\
& =p_{x}(\lambda)
\end{aligned}
$$

Hence, we show that $i d_{X}:\left(X, \tau_{\Theta_{\tau_{X}}}\right) \rightarrow\left(X, \tau_{X}\right)$ is continuous.

Therefore, $\tau:$ FYAPP $\rightarrow$ FYPT is a left adjoint of $\Theta:$ FYPT $\rightarrow$ FYAPP.

Now we show that an Alexandroff $L$-fuzzifying topology can be induced from an Alexandroff $L$-fuzzifying pretopology in the following manner.

Lemma 3.1 [10] Let $(X, \theta)$ be an L-fuzzifying approximation space based on $L$-fuzzy preorder relation $\theta$. Then $p_{x}^{\theta}$ defined in Equation (1), satisfies

$$
p_{x}^{\theta}(\lambda)=\bigvee_{x \in \mu \subseteq \lambda} \bigwedge_{y \in \mu} p_{y}^{\theta}(\mu)
$$

Remark 3.2 An L-fuzzifying pretopology $\tau_{\theta}=\left\{p_{x}^{\theta}\right.$ : $\mathcal{P}(X) \rightarrow L \mid x \in X\}$ which satisfies Lemma 3.1, is called topological.

Proposition 3.9 Let $(X, \theta)$ be an L-fuzzifying approximation space and $\theta$ be $L$-fuzzy preorder relation. Then for all $\lambda \in \mathcal{P}(X), \mathcal{T}^{\theta}: \mathcal{P}(X) \rightarrow L$ defined as following

$$
\mathcal{T}^{\theta}(\lambda)=\bigwedge_{x \in \lambda} p_{x}^{\theta}(\lambda)(x)
$$

is an Alexandroff L-fuzzifying topology on $X$.

Proof: To prove the result we need to show that $\mathcal{T}^{\theta}$, satisfies the properties (i), (iii) and (iv) listed in Definition 2.4 .

(i) Since from Equation $(1),\left(p_{x}^{\theta}(\lambda)=\underline{\theta}(\lambda)(x)\right)$. Hence, $\mathcal{T}^{\theta}(\phi)=\mathcal{T}^{\theta}(X)=1$, can be easily verified by the definition of $\underline{\theta}$. (iii) For each $\left\{\lambda_{i} \mid i \in I\right\} \subseteq \mathcal{P}(X)$,

$$
\begin{aligned}
\mathcal{T}^{\theta}\left(\bigcap_{i \in I} \lambda_{i}\right) & =\bigwedge_{x \in \cap_{i \in I} \lambda_{i}} p_{x}^{\theta}\left(\bigcap_{i \in I} \lambda_{i}\right)(x) \\
& =\bigwedge_{x \in \cap_{i \in I} \lambda_{i}} \bigwedge_{i \in I} p_{x}^{\theta}\left(\lambda_{i}\right)(x) \\
& =\bigwedge_{i \in I} \bigwedge_{x \in \cap_{i \in I} \lambda_{i}} p_{x}^{\theta}\left(\lambda_{i}\right)(x) \\
& \geq \bigwedge_{i \in I} \bigwedge_{x \in \lambda_{i}} p_{x}^{\theta}\left(\lambda_{i}\right)(x) \\
& =\bigwedge_{i \in I} \mathcal{T}^{\theta}\left(\lambda_{i}\right) .
\end{aligned}
$$

(iv) For each $\left\{\lambda_{i} \mid i \in I\right\} \subseteq \mathcal{P}(X)$,

$$
\begin{aligned}
\mathcal{T}^{\theta}\left(\bigcup_{i \in I} \lambda_{i}\right) & =\bigwedge_{x \in \cup_{i \in I} \lambda_{i}} p_{x}^{\theta}\left(\bigcup_{i \in I} \lambda_{i}\right)(x) \\
& =\bigwedge_{i \in I} \bigwedge_{x \in \lambda_{i}} p_{x}^{\theta}\left(\bigcup_{i \in I} \lambda_{i}\right)(x) \\
& \geq \bigwedge_{i \in I} \bigwedge_{x \in \lambda_{i}} p_{x}^{\theta}\left(\lambda_{i}\right)(x) \\
& =\bigwedge_{i \in I} \mathcal{T}^{\theta}\left(\lambda_{i}\right) .
\end{aligned}
$$

Hence, $\mathcal{T}^{\theta}$ is an Alexandroff $L$-fuzzifying topology on $X$.

\section{Conclusion}

This paper contributes to the theory of $L$-fuzzifying topology originated from [20]. We have shown that an $L$-fuzzifying pretopology can be generated by a reflexive $L$-fuzzy relation using the concept of lower $L$-fuzzifying approximation operator. Further, we have established the adjointness between category of $L$-fuzzifying approximation spaces based on reflexive $L$-fuzzy relations and $L$-fuzzifying pretopological spaces. Moreover, we proved that if we consider an $L$-fuzzifying approximation space with $L$-fuzzy preorder, then we can induce an Alexandroff $L$ fuzzifying topology from an Alexandroff $L$-fuzzifying pretopology.

\section{Acknowledgment:}

The work of the first author was supported by University of Ostrava grant IRP201824 "Complex topological structures" and the work of Irina Perfilieva was partially supported by the project AI-Met4AI, CZ.02.1.01/0.0/0.0/17-049/0008414.

\section{References}

[1] J. Adámek, H. Herrlich, G. Strecker, Abstract and concrete categories. 
[2] D. Boixader, J. Jacas, J. Recasens, Upper and lower approximations of fuzzy sets, International Journal of General System 29 (2000) 555-568.

[3] D. Dubois, H. Prade, Rough fuzzy sets and fuzzy rough sets, International Journal of General System 17 (1990) 191-209.

[4] J. Fang, P. Chen, One-to-one correspondence between fuzzifying topologies and fuzzy preorders, Fuzzy Sets and Systems 158 (2007) 1814-1822.

[5] J. Fang, Y. Qiu, Fuzzy orders and fuzzifying topologies, International Journal of Approximate Reasoning 48 (2008) 98-109.

[6] J. A. Goguen, L-fuzzy sets, Journal of Mathematical Analysis and Applications 18 (1967) 145-174.

[7] J. Hao, Q. Li, The relationship between L-fuzzy rough set and L-topology, Fuzzy Sets and Systems 178 (2011) 74-83.

[8] H. Lai, D. Zhang, Fuzzy preorder and fuzzy topology, Fuzzy Sets and Systems 157 (2006) 18651885 .

[9] R. Lowen, L. Xu, Alternative characterizations of FNCS, Fuzzy Sets and Systems 104 (1999) 381391.

[10] B. Pang, J. S. Mi, Z. Y. Xiu, L-fuzzifying approximation operators in fuzzy rough sets, Information Sciences 480 (2019) 14-33.

[11] Z. Pawlak, Rough sets, International Journal of Computer \& Information Sciences 11 (1982) 341356.

[12] I. Perfilieva, A. P. Singh, S. P. Tiwari, On Ftransforms, L-fuzzy partitions and L-fuzzy pretopological spaces, in: Computational Intelligence (SSCI), 2017 IEEE Symposium Series on, IEEE, 2017, pp. 1-8.

[13] I. Perfilieva, A. P. Singh, S. P. Tiwari, On the relationship among F-transform, fuzzy rough set and fuzzy topology, Soft Computing 21 (2017) 35133523 .

[14] I. Perfilieva, S. P. Tiwari, A. P. Singh, Latticevalued $\mathrm{F}$-transforms as interior operators of $\mathrm{L}$ fuzzy pretopological spaces, in: International Conference on Information Processing and Management of Uncertainty in Knowledge-Based Systems, 2018, pp. 163-174.

[15] J. Qiao, B. Q. Hu, A short note on L-fuzzy approximation spaces and L-fuzzy pretopological spaces, Fuzzy Sets and Systems 312 (2017) 126134.
[16] K. Qin, Z. Pei, On the topological properties of fuzzy rough sets, Fuzzy Sets and Systems 151 (2005) 601-613.

[17] Y. H. She, G. J. Wang, An axiomatic approach of fuzzy rough sets based on residuated lattices, Computers \& Mathematics with Applications 58 (2009) 189-201.

[18] S. P. Tiwari, A. K. Srivastava, Fuzzy rough sets, fuzzy preorders and fuzzy topologies, Fuzzy sets and systems 210 (2013) 63-68.

[19] W. Yao, F. G. Shi, A note on specialization L-preorder of L-topological spaces, Lfuzzifying topological spaces, and L-fuzzy topological spaces, Fuzzy Sets and Systems 159 (2008) 2586-2595.

[20] M. Ying, A new approach for fuzzy topology (i), Fuzzy sets and Systems 39 (1991) 303-321.

[21] L. A. Zadeh, Similarity relations and fuzzy orderings, Information sciences 3 (1971) 177-200.

[22] D. Zhang, Fuzzy pretopological spaces, an extensional topological extension of FTS, Chinese Annals of Mathematics 20 (1999) 309-316.

[23] D. Zhang, L-fuzzifying topologies as L-topologies, Fuzzy Sets and Systems 125 (2002) 135-144.

[24] D. Zhang, L. Xu, Categories isomorphic to FNS, Fuzzy Sets and Systems 104 (1999) 373-380. 